\title{
Synthesis of Phenolic Compounds by Trapping Arynes with a Hydroxy Surrogate
}

\author{
Rajdip Karmakar ${ }^{1}$, Sourav Ghorai ${ }^{1}$, Yuanzhi Xia ${ }^{2}$ and Daesung Lee ${ }^{1, *}$ \\ 1 Department of Chemistry, University of Illinois at Chicago, 845 West Taylor Street, Chicago, \\ IL 60607, USA; E-Mails: rkarma2@uic.edu (R.K.); sghora2@uic.edu (S.G.) \\ 2 College of Chemistry and Materials Engineering, Wenzhou University, Wenzhou 325035, \\ Zhejiang, China; E-Mail: xyz@wzu.edu.cn \\ * Author to whom correspondence should be addressed; E-Mail: dsunglee@uic.edu; \\ Tel.: +1-312-996-5189; Fax: +1-312-996-0431.
}

Academic Editor: Hiroto Yoshida

Received: 6 August 2015 / Accepted: 24 August 2015 / Published: 31 August 2015

\begin{abstract}
Trapping of arynes with various nucleophiles provides a range of heteroatom-functionalized arene derivatives, but the corresponding reaction with water does not provide phenol derivatives. Silver trifluroacetate $\left(\mathrm{AgO}_{2} \mathrm{CCF}_{3}\right)$ can nicely solve this problem. It was found that in typical organic solvent, $\mathrm{AgO}_{2} \mathrm{CCF}_{3}$ readily reacts with arynes to generate trifluoroacetoxy organosilver arene intermediate, which, upon treating with silica gel, provides phenolic products. This protocol can be extended to the synthesis of $\alpha$-halofunctionalized phenol derivatives by simply adding NBS ( $N$-bromosuccinimides) or NIS ( $N$-iodosuccinimides) to the reaction along with silver trifluroacetate, which provided $\alpha$-bromo or $\alpha$-iodophenol derivatives in good yield. However, the similar reactions with NCS ( $N$-chlorosuccinimides) afforded only the protonated product instead of the expected $\alpha$-chlorophenols derivatives. Interestingly, substrates containing silyl substituents on 1,3-diynes resulted in $\alpha$-halotrifluoroacetates rather than their hydrolyzed product. Additionally, trapping the same arynes with other oxygen-based nucleophiles containing silver counter cation, along with NXS ( $N$-halosuccinimides), generated $\alpha$-halooxyfunctionalized products.
\end{abstract}

Keywords: aryne; bis-functionalization; halophenol; silver trifluoroacetate; regioselectivity 


\section{Introduction}

A variety of trapping reactions of arynes [1-8] have been reported on the basis of their highly electrophilic nature [9]. In contrast, a brief screening of literature readily identifies the lack of the examples of aryne trapping with water under traditional aryne formation conditions [10-16] or under the conditions of the hexadehydro Diels-Alder reaction [17-20]. Although, in theory, water should be a suitable nucleophile to react with arynes similar to alcohols and carboxylic acids [21,22], the lack of successful trapping of arynes with water might be the consequence of the immiscibility of water with the transient arynes generated in organic solvent, typically $\mathrm{CH}_{2} \mathrm{Cl}_{2}$ or toluene.

It would be highly desirable if we could expand the aryne trapping reaction to directly install a phenolic hydroxyl group on arene scaffolds, as this is an important functionality in large number of compounds, including natural products and pharmaceuticals $[23,24]$. In search of suitable reagents that can behave like a water surrogate under the given reaction conditions, we refer to a clue suggested by our previous nucleophile trapping study [21,25] of arynes, formed from various tetraynes (1), where nucleophiles $\left(\mathrm{F}^{-}, \mathrm{F}_{3} \mathrm{C}^{-}, \mathrm{CF}_{3} \mathrm{~S}^{-}\right)$associated with a silver counter cation, including silver trifluroacetate $\left(\mathrm{AgO}_{2} \mathrm{CCF}_{3}\right)$, and provided excellent yields of the corresponding adducts (Scheme 1). Surprisingly, for the similar reaction with silver trifluoroacetate, the protonation of the initially formed putative intermediate $\mathbf{2}$ did not lead to the expected trifluoroacetate 3 , instead, its deacetylated phenolic product $\mathbf{4}$ was obtained after purification [21].

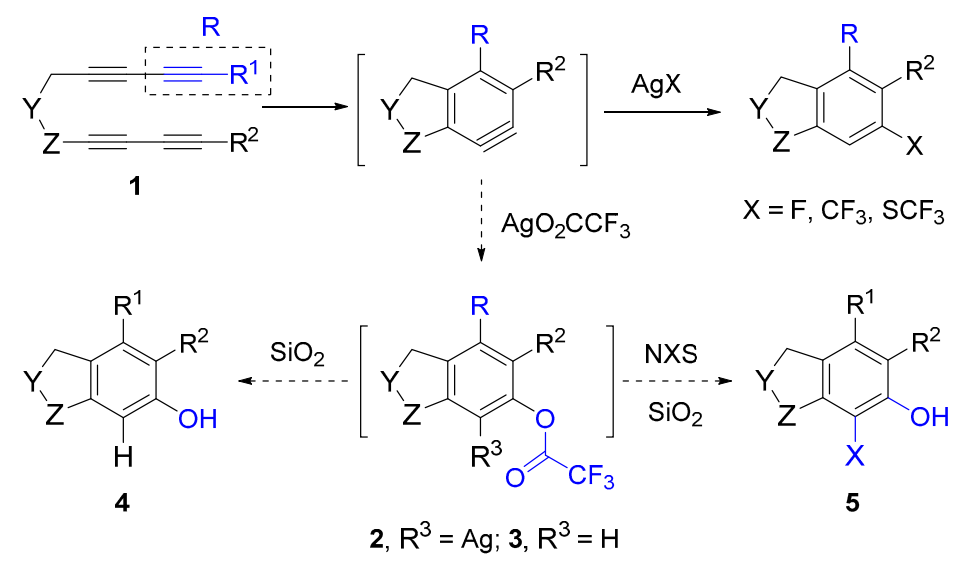

Scheme 1. Trapping reactions of an in situ generated aryne intermediate with various nucleophiles with a silver counter cation.

\section{Results and Discussion}

On the basis of this initial observation, we carried out a systematic study of aryne trapping reactions with $\mathrm{AgO}_{2} \mathrm{CCF}_{3}$ as a water surrogate to prepare a variety of highly functionalized arene products containing a free phenolic hydroxyl group, and, herein, we report the results.

First, reactions with both symmetrical and unsymmetrical tetrayne substrates of varying substituents were screened to optimize conditions that produce formal water addition products (Table 1). It was quickly identified that the reaction with 1.5 equivalents of $\mathrm{AgO}_{2} \mathrm{CCF}_{3}$ in toluene at $90{ }^{\circ} \mathrm{C}$, followed by purification on silica gel, afforded the phenolic products in good yields. Oxygen-tethered symmetrical tetrayne 1a with butyl substituents provided a mixture of ortho- and meta-OH adducts $\boldsymbol{o}-\mathbf{4 a}$ and $\boldsymbol{m}-\mathbf{- 4 a}$ 
in a 1.3:1 ratio (Entry 1). The reaction of all-carbon tethered substrate $\mathbf{1 b}$ with a gem-dicarboxylate moiety in place of the oxygen tether afforded a similar result, but with slightly improved selectivity and yield (87\%) of $\boldsymbol{o}-\mathbf{4 b}$ and $\boldsymbol{m}-\mathbf{4 b}$ (Entry 2) [26]. Replacing the butyl groups with trimethylsilyl groups afforded only a single isomer o-4c (Entry 3) [21,27-37]. Although the tether was also changed from oxygen in 1a to tosylated nitrogen in 1c, we believe this change has negligible impact on the selectivity. As expected, an ynamide-tethered unsymmetrical tetrayne with triethylsilyl substituents 1d afforded only the ortho isomer $\boldsymbol{o - 4 d}$ in $66 \%$ yield (Entry 4 ). A complete switch in regioselectivity was observed when a tosylated nitrogen tethered symmetrical bis-1,3-diyne with phenyl substituents was used, which provided in a majority $\boldsymbol{m}-\mathbf{4 e}$ along with $\boldsymbol{o - 4 e}$ in a 6.6:1 ratio (entry 5). This switch in regioselectivity can be explained in terms of the charge-controlled model [30], where the electron withdrawing phenyl group creates a more positive character on the farther carbon of the aryne. This allows the nucleophile to attack the meta carbon more preferably. This clearly indicates that, not the tether, but the substituents at the terminal carbon of the 1,3-diyne moieties are the main controlling elements for the selectivity [38].

Table 1. Trapping reactions of various aryne intermediate to form phenolic products.
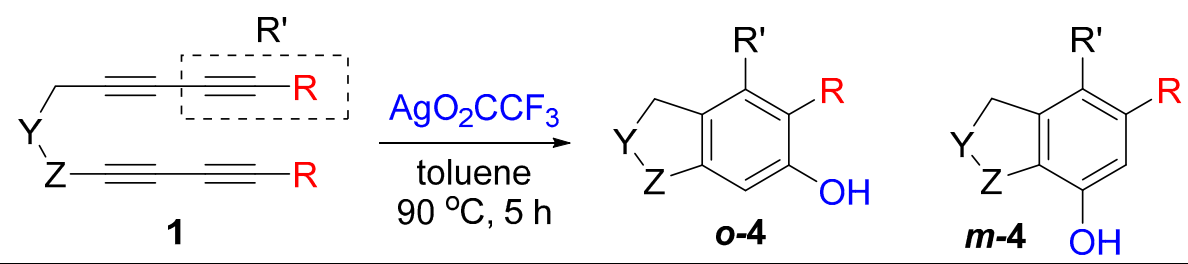

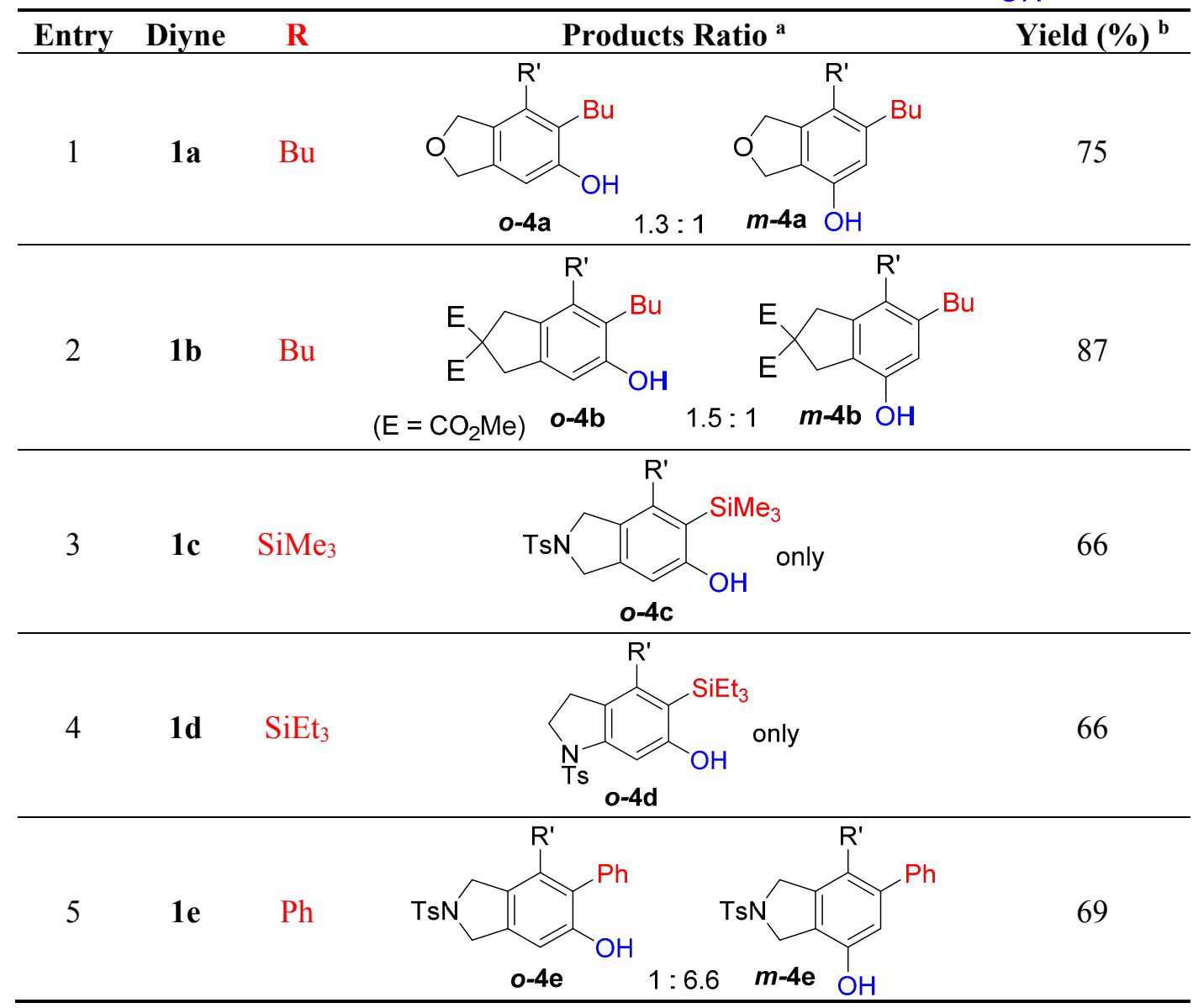

a The ratio was determined with the isolated product. ${ }^{\mathrm{b}}$ Isolated yield after $\mathrm{SiO}_{2}$ chromatography. 
With this result in hand, we envisioned that the putative organosilver intermediate 2 might be captured by suitable electrophiles to generate $\alpha$-functionalized phenol derivatives. To test the viability of this hypothesis, the reaction was run with $N$-halosuccinimides under otherwise identical conditions, and the results are summarized in Table 2.

Table 2. 1,2-Bis functionalization to form $\alpha$-halophenol derivatives.

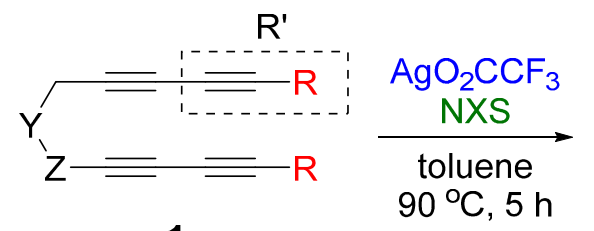<smiles>[Y]Cc1c([R])c([R])c(O)c([X])c1[R]</smiles><smiles>[Y]Cc1c([R])c([R])c(O)c([X])c1[Y]</smiles>

Entry Diyne

a The ratio was determined with the isolated product. ${ }^{\mathrm{b}}$ Isolated yield after $\mathrm{SiO}_{2}$ chromatography.

When substrate 1a was treated with $\mathrm{AgO}_{2} \mathrm{CCF}_{3}$ (1.5 equiv.) and NBS (2.0 equiv.), a mixture of $\alpha$-bromophenol derivatives $\boldsymbol{o - 5 a - B r}$ and $\boldsymbol{m}$-5a-Br were obtained in $69 \%$ yield with a $1.6: 1$ ratio (Entry 1 ). Similarly, with NIS instead of NBS, the corresponding $\alpha$-iodophenol derivatives $\boldsymbol{o}$-5a-I and $\boldsymbol{m}$-5a-I were isolated in 67\% yield with a 1.8:1 ratio (Entry 2). Substrate $\mathbf{1 b}$ furnished the bromophenol derivatives in $63 \%$ yield with an expected selectivity of 1.4:1 [26]. $N$-Tosylamide tethered substrate 1f containing 
$n$-hexyl substituents provided bromo and iodophenol derivatives $\boldsymbol{o - 5 f}-\mathbf{B r} / \boldsymbol{m}-\mathbf{5 f}-\mathbf{B r}$ and $\boldsymbol{o - 5 a - I} / \boldsymbol{m}-\mathbf{5 a}-\mathbf{I}$ in $83 \%$ and $88 \%$ yield with a 1.8 and 2.6 ratio, respectively (Entries 5 and 6 ). Tetrayne 1 e containing phenyl substituents was found to be less efficient and provided a mixture of $\boldsymbol{o - 5 e - B r}$ and $\boldsymbol{m}-\mathbf{5 e}-\mathbf{B r}$ in only $36 \%$ yield (Entry 4 ).

While exploring the scope of the direct synthesis of $\alpha$-halophenol derivatives, we found that the silyl substituent ortho to the trifluoroacetate moiety interferes with its hydrolysis when halogen was incorporated. Thus, the reaction of $\mathbf{1 g}$ afforded single regioisomer $\boldsymbol{o}-\mathbf{6} \mathbf{g}-\mathbf{C F}_{\mathbf{3}}$ as a major product along with expected phenolic product $\mathbf{o - 5 g - B r}$ in $10 \%$ yield (Entry 1) (Table 3 ). This is in stark contrast to the formation of $\boldsymbol{o}-\mathbf{4 c}$ and $\boldsymbol{o - 4 d}$, which are derived from their precursors via complete hydrolysis of the corresponding trifluoroacetates. Based on this observation, we further explored the 1,2-oxyhalogenation to form oxygen-masked form of halophenol derivatives (Table 3). The reaction of substrate 1c in the presence of silver acetate and NBS provided single regioisomer $\boldsymbol{o}-\mathbf{6 c -}-\mathbf{C H}_{3}$ along with phenolic product $\boldsymbol{o - 5 c - B r}$ in a 1:2.2 ratio (Entry 2). Unexpectedly, however, the reaction of 1c with $\mathrm{AgO}_{2} \mathrm{CCF}_{3}$ and NIS afforded a mixture of iodotrifluoroacetates $\boldsymbol{o - 6} \mathbf{c}$ and $\boldsymbol{m}-\mathbf{6 c}$ in a 6.2:1 ratio devoid of hydrolyzed product (Entry 3 ). Substrates 1a and $\mathbf{1 f}$ upon treating with silver triflate and NBS afforded a mixture of bromotriflates $\boldsymbol{o - 6 a} / \boldsymbol{m}$-6a $(2.6: 1)$ and $\boldsymbol{o - 6 f} / \boldsymbol{m}$-6f $(4.8: 1)$ in $82 \%$ and $94 \%$ yield, respectively (Entries 4 and 5) [39]. The reaction of $\mathbf{1 f}$ with silver benzoate and NBS provided a mixture of $\alpha$-bromobenzoates $\boldsymbol{o}-\mathbf{6 f - B r}$ and $\boldsymbol{m}-\mathbf{6 f - B r}$ in 30\% yield with a 2:1 ratio (Entry 6), but, with NCS, not even traces of the expected chloride-trapped product were obtained, instead only protonated products $\boldsymbol{o}-\mathbf{6 f}-\mathbf{H}$ and $\boldsymbol{m}-\mathbf{6 f}-\mathbf{H}$ were isolated in $76 \%$ yield with a 2.1:1 ratio (Entry 7 ).

Table 3. 1,2-Bis functionalization to form oxygen-masked $\alpha$-halophenol derivatives.

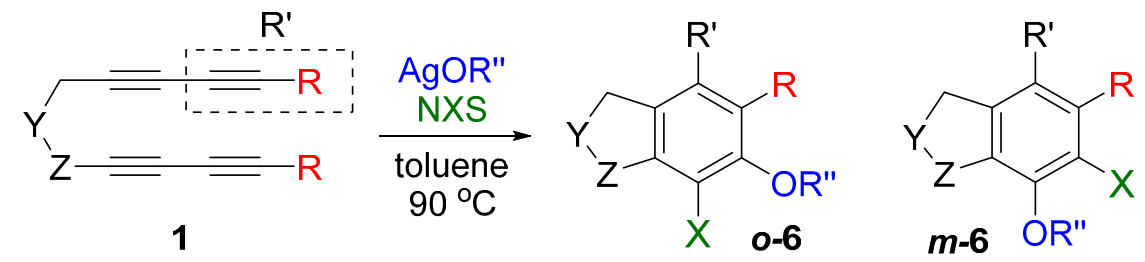

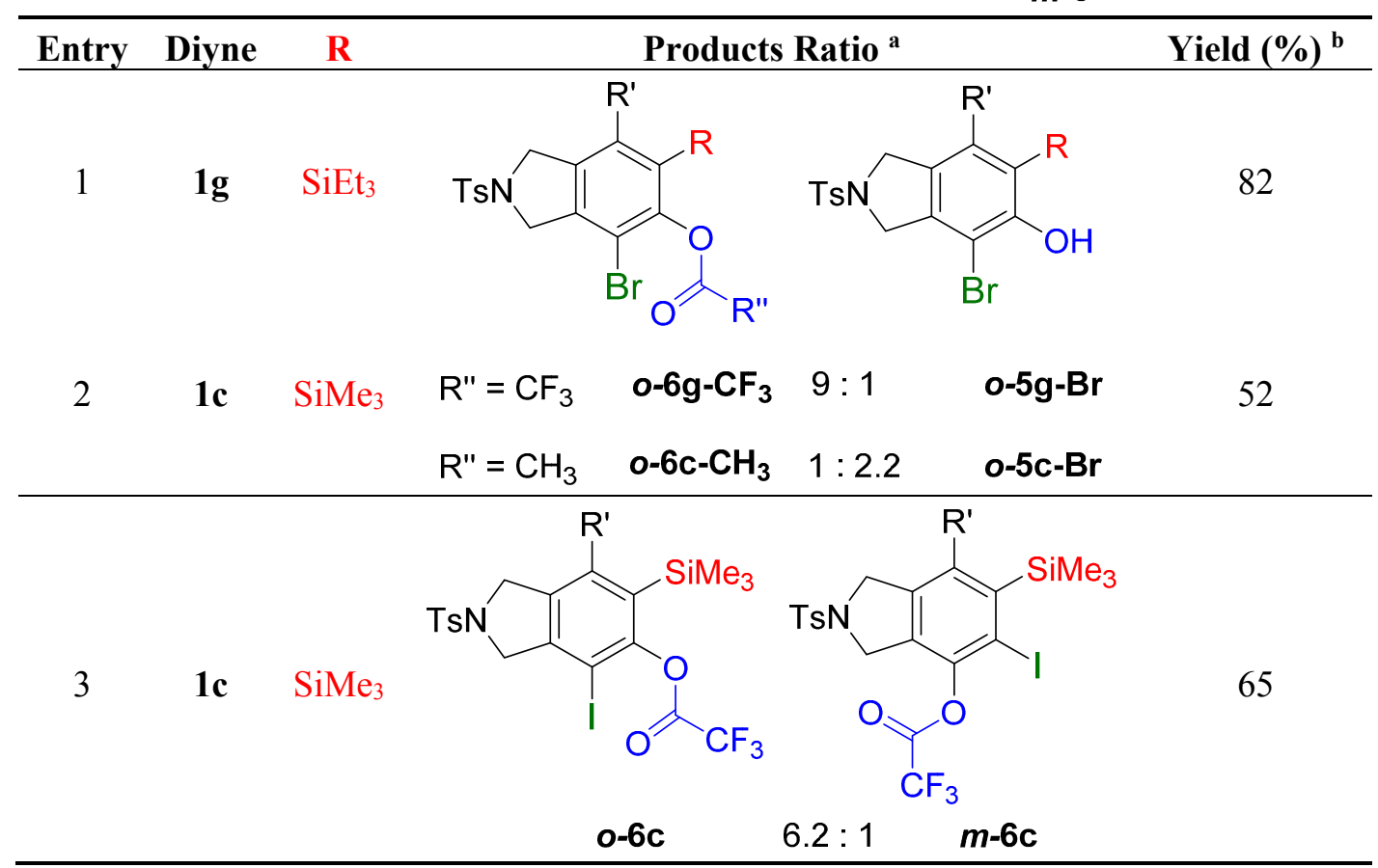


Table 3. Cont.

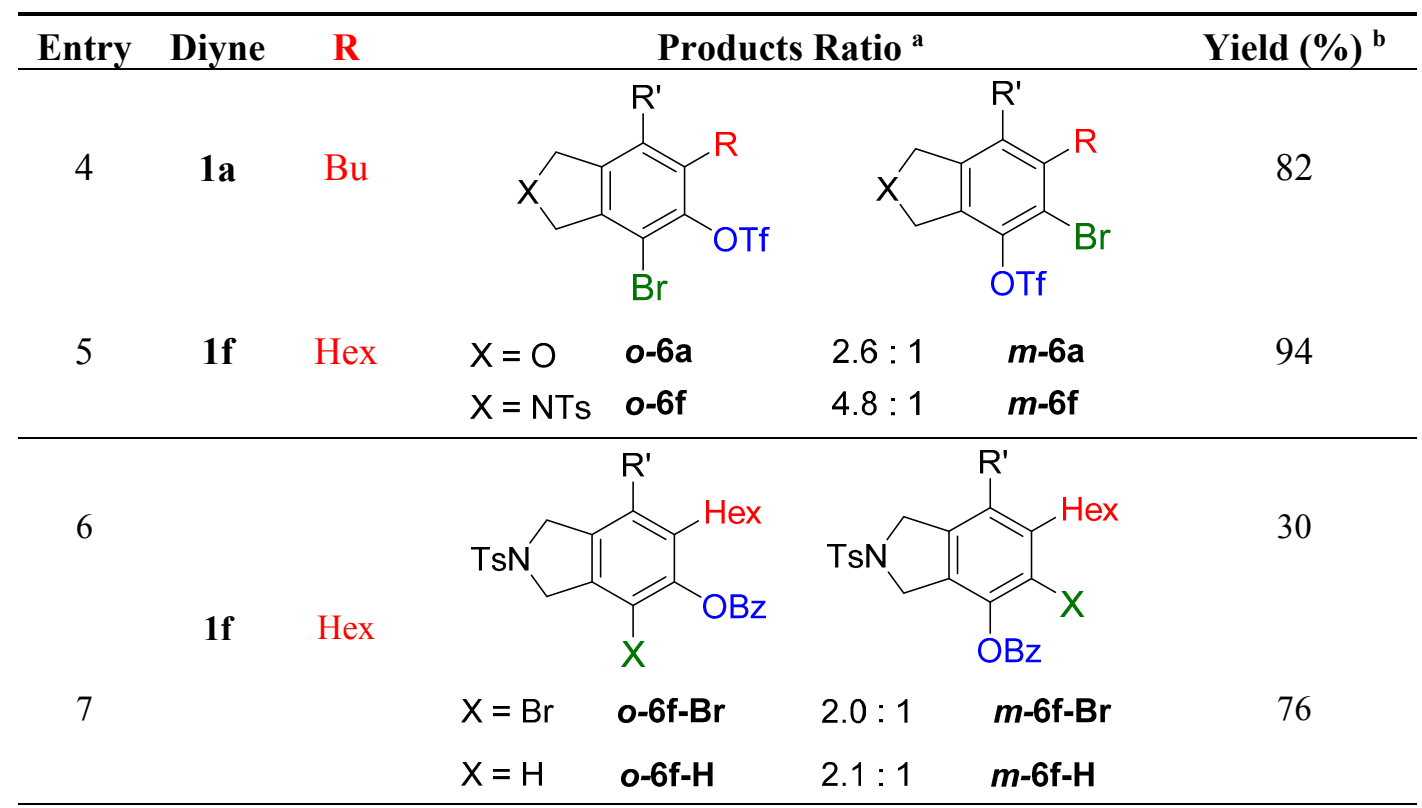

a The ratio was determined with the isolated product. ${ }^{\mathrm{b}}$ Isolated yield after $\mathrm{SiO}_{2}$ chromatography.

\section{Experimental Section}

\subsection{General Information}

Reactions were carried out in oven-dried glassware unless otherwise noted. Compounds were purchased from Aldrich, Acros, TCI America, or Oakwood Chemicals, unless otherwise noted. Toluene, acetonitrile, and dichloromethane were distilled over calcium hydride $\left(\mathrm{CaH}_{2}\right)$ under a nitrogen atmosphere. THF was distilled over sodium-benzophenone ketyl under a nitrogen atmosphere. Column chromatography was performed using silica gel $60 \AA$ (32-63 mesh), purchased from Silicycle Inc. (Quebec, QC, Canada). Analytical thin layer chromatography (TLC) was performed on $0.25 \mathrm{~mm}$ E. Merck precoated silica gel 60 (particle size $0.040-0.063 \mathrm{~mm}$ ). Yields refer to chromatographically and spectroscopically pure compounds unless otherwise stated. ${ }^{1} \mathrm{H}-\mathrm{NMR}$ and ${ }^{13} \mathrm{C}-\mathrm{NMR}$ spectra were recorded on a Bruker AV-500 spectrometer (Bruker BioSpin Corporation, Billerica, MA, USA). ${ }^{19}$ F-NMR spectrum was recorded in Varian Mercury-Vx-300 spectrometer (Palo Alto, CA, USA). ${ }^{1} \mathrm{H}-\mathrm{NMR}$ chemical shifts $(\delta)$ are reported in parts per million (ppm) downfield of TMS and are referenced relative to the residual proteated solvent peak $\left(\mathrm{CDCl}_{3}(7.26 \mathrm{ppm})\right) .{ }^{13} \mathrm{C}$ chemical shifts $(\delta)$ are reported in parts per million downfield of TMS and are referenced to the carbon resonance of the solvent $\left(\mathrm{CDCl}_{3}(77.2 \mathrm{ppm})\right)$. Multiplicities are indicated by s (singlet), d (doublet), $\mathrm{t}$ (triplet), q (quartet), quin (quintet), sext (sextet), or m (multiplet). ${ }^{1} \mathrm{H}-\mathrm{NMR}$ signals that fall within a $c a$. 0.3 -ppm range are generally reported as a multiplet, with a range of chemical shift values corresponding to the peak or center of the peak. Coupling constants, $J$, are reported in $\mathrm{Hz}$ (Hertz). Electrospray ionization (ESI) mass spectra were recorded on a Waters Micromass Q-Tof Ultima (Waters Corporation, Milford, MA, USA) at the University of Illinois at Urbana-Champaign. Electron impact (EI) mass spectra and Chemical Ionization (CI) mass spectra were obtained using a Micromass 70-VSE (Waters Corporation, Milford, MA, USA) at the University of Illinois at Urbana-Champaign. 


\subsection{Experimental Details}

\subsubsection{General Procedure for the Mono-Functionalization (GPM)}

In a glove box, a mixture of a substrate $(0.1 \mathrm{mmol}, 1.0$ equiv. $)$ and a nucleophile $(0.15 \mathrm{mmol}$, 1.5 equiv.) in dry toluene $(3 \mathrm{~mL})$ was taken into a Schlenk tube. The reaction mixture was stirred at $90{ }^{\circ} \mathrm{C}$ for $5 \mathrm{~h}$, unless otherwise noted. After completion, the reaction mixture was transferred to a round-bottom flask, concentrated and loaded on silica gel column for chromatographic purification, using ethyl acetate-hexane mixture as the eluent.

\subsubsection{General Procedure for the Bis-Functionalization (GPB)}

In a glove box, a mixture of a substrate $(0.1 \mathrm{mmol}, 1.0$ equiv. $)$ and a nucleophile $(0.15 \mathrm{mmol}$, 1.5 equiv.) and an electrophile $(0.2 \mathrm{mmol}, 2.0$ equiv. $)$ in dry toluene $(3 \mathrm{~mL})$ was taken into a Schlenk tube. The reaction mixture was stirred at $90{ }^{\circ} \mathrm{C}$ for $5 \mathrm{~h}$, unless otherwise noted. After completion, the reaction mixture was transferred to a round-bottom flask, concentrated and subjected to column chromatography, using ethyl acetate-hexane mixture as the eluent, to get pure products.

3.2.3. Characterization Data of the Products<smiles>Oc1cc2c(c(C#CBr)c1Br)COC2</smiles>

$\boldsymbol{o - 4 a}$ : This compound was prepared using GPM as an inseparable mixture of isomers $(\mathrm{o} / \mathrm{m}=1.3: 1)$ in $75 \%$ overall yield after column chromatographic purification. ${ }^{1} \mathrm{H}-\mathrm{NMR}\left(\mathrm{CDCl}_{3}, 500 \mathrm{MHz}\right): \delta$ (major isomer) $6.58(\mathrm{~s}, 1 \mathrm{H}), 5.07-5.04(\mathrm{~m}, 4 \mathrm{H}), 4.76(\mathrm{~s}, 1 \mathrm{H}), 2.78(\mathrm{t}, 2 \mathrm{H}, J=8.0 \mathrm{~Hz}), 2.45(\mathrm{t}, 2 \mathrm{H}, J=7.0 \mathrm{~Hz})$, 1.64-1.36 (m, 8H), 0.97-0.92 (m, 6H); ${ }^{13} \mathrm{C}-\mathrm{NMR}\left(\mathrm{CDCl}_{3}, 125 \mathrm{MHz}\right): \delta$ (major isomer) 153.1, 137.1, 134.0, 129.6, 118.2, 107.4, 97.5, 76.6, 74.1, 74.0, 31.7, 30.9, 27.8, 22.9, 21.9, 19.2, 14.0, 13.6; HRMS (ESI) calcd for $\mathrm{C}_{18} \mathrm{H}_{25} \mathrm{O}_{2}[\mathrm{M}+\mathrm{H}]^{+} 273.1849$, found 273.1850 .

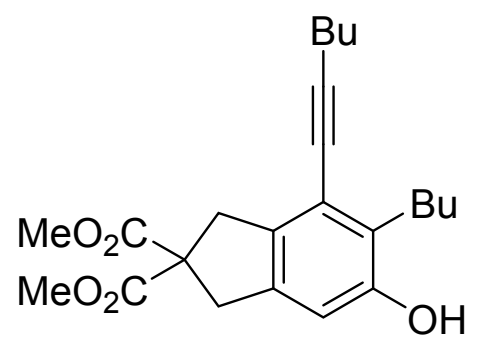

$\boldsymbol{o - 4 b}$ : This compound was formed through GPM at $120{ }^{\circ} \mathrm{C}$ (overnight) in an inseparable mixture of two isomers with $66 \%$ overall yield after purification by column chromatography. ${ }^{1} \mathrm{H}-\mathrm{NMR}\left(\mathrm{CDCl}_{3}\right.$, $500 \mathrm{MHz}): \delta$ (major isomer) $6.55(\mathrm{~s}, 1 \mathrm{H}), 4.71(\mathrm{~s}, 1 \mathrm{H}), 3.75(\mathrm{~s}, 6 \mathrm{H}), 3.57(\mathrm{~s}, 2 \mathrm{H}), 3.51(\mathrm{~s}, 2 \mathrm{H}), 2.73$ $(\mathrm{t}, 2 \mathrm{H}, J=7.8 \mathrm{~Hz}), 2.46(\mathrm{t}, 2 \mathrm{H}, J=7.0 \mathrm{~Hz}), 1.62-1.32(\mathrm{~m}, 8 \mathrm{H}), 0.99-0.89(\mathrm{~m}, 6 \mathrm{H}) ;{ }^{13} \mathrm{C}-\mathrm{NMR}\left(\mathrm{CDCl}_{3}\right.$, 
$125 \mathrm{MHz}$ ): $\delta$ (all discernible signals for both isomers) 172.3, 172.1, 152.8, 150.7, 145.9, 144.8, 137.7, 134.6, 129.2, 122.7, 120.8, 114.2, 112.2, 110.6, 110.0, 97.4, 95.8, 59.8, 59.6, 53.0, 53.0, 41.3, 40.8, $40.4,37.3,34.0,32.9,31.7,31.1,31.0,28.0,22.9,22.6,22.0,19.3,14.0,13.95,13.6$; HRMS (ESI) calcd for $\mathrm{C}_{23} \mathrm{H}_{31} \mathrm{O}_{5}[\mathrm{M}+\mathrm{H}]^{+} 387.2166$, found 387.2162.

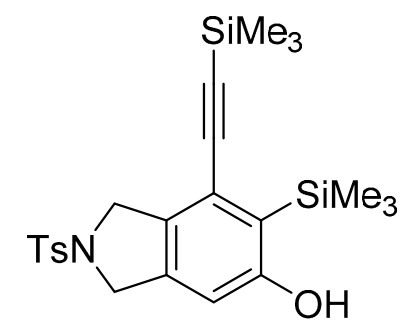

$\boldsymbol{o - 4 c}$ : This compound was prepared using GPM. only single isomer was isolated in $66 \%$ after purification using column chromatography. ${ }^{1} \mathrm{H}-\mathrm{NMR}\left(\mathrm{CDCl}_{3}, 500 \mathrm{MHz}\right) \delta$ 7.77-7.74 $(\mathrm{m}, 2 \mathrm{H}), 7.33-7.30$ $(\mathrm{m}, 2 \mathrm{H}), 6.49(\mathrm{~s}, 1 \mathrm{H}), 5.28(\mathrm{~s}, 1 \mathrm{H}), 4.55(\mathrm{~s}, 4 \mathrm{H}), 2.41(\mathrm{~s}, 3 \mathrm{H}), 0.41(\mathrm{~s}, 9 \mathrm{H}), 0.25(\mathrm{~s}, 9 \mathrm{H}) ;{ }^{13} \mathrm{C}-\mathrm{NMR}\left(\mathrm{CDCl}_{3}\right.$, $125 \mathrm{MHz}): \delta 160.57,143.7,138.3,133.9,132.8,129.8,127.6,125.4,124.8,109.8,103.4,103.0,54.3$, 53.8, 21.5, 1.1, -0.3; HRMS (ESI) calcd for $\mathrm{C}_{23} \mathrm{H}_{32} \mathrm{NO}_{3} \mathrm{SSi}_{2}[\mathrm{M}+\mathrm{H}]^{+} 458.1636$, found 458.1624 .<smiles>CCC[SiH2]C#Cc1c(O)cc2c(c1[SiH2]CC)CCN2C</smiles>

$\boldsymbol{o - 4 d}$ : This compound was prepared using GPM and isolated in 66\% yield as a single isomer after column chromatographic purification. ${ }^{1} \mathrm{H}-\mathrm{NMR}\left(\mathrm{CDCl}_{3}, 500 \mathrm{MHz}\right): \delta 7.73-7.69(\mathrm{~m}, 2 \mathrm{H}), 7.28-7.24$ $(\mathrm{m}, 2 \mathrm{H}), 7.02(\mathrm{~s}, 1 \mathrm{H}), 5.09(\mathrm{~s}, 1 \mathrm{H}), 3.88(\mathrm{t}, 2 \mathrm{H}, J=10 \mathrm{~Hz}), 2.92(\mathrm{t}, 2 \mathrm{H}, J=10 \mathrm{~Hz}), 2.39(\mathrm{~s}, 3 \mathrm{H})$, 1.03-0.91 (m, 24H), 0.67-0.57 (m, 6H); ${ }^{13} \mathrm{C}-\mathrm{NMR}\left(\mathrm{CDCl}_{3}, 125 \mathrm{MHz}\right): \delta 161.6,144.3,143.5,134.0$, 129.8, 128.6, 127.3, 126.7, 117.2, 104.8, 102.3, 99.9, 49.9, 27.9, 21.6, 7.7, 7.4, 4.7, 4.3; HRMS (ESI) calcd for $\mathrm{C}_{29} \mathrm{H}_{44} \mathrm{NO}_{3} \mathrm{SSi}_{2}[\mathrm{M}+\mathrm{H}]^{+} 542.2575$, found 542.2571 .<smiles>Oc1cc2c(c(C#Cc3ccccc3)c1-c1ccccc1)CN([Te])C2</smiles>

o-4e: This compound was produced through GPM with other isomer with $69 \%$ overall yield after purification by column chromatography. ${ }^{1} \mathrm{H}-\mathrm{NMR}\left(\mathrm{CDCl}_{3}, 500 \mathrm{MHz}\right): \delta$ (major isomer) 7.83-7.80 (m, 2H), 7.59-7.55 (m, 2H) 7.43-7.28 (m, 10H), $6.74(\mathrm{~s}, 1 \mathrm{H}), 5.74(\mathrm{~s}, 1 \mathrm{H}), 4.79(\mathrm{~m}, 2 \mathrm{H}), 4.69(\mathrm{~m}, 2 \mathrm{H})$, $2.41(\mathrm{~s}, 3 \mathrm{H}) ;{ }^{13} \mathrm{C}-\mathrm{NMR}\left(\mathrm{CDCl}_{3}, 125 \mathrm{MHz}\right): \delta$ (major isomer) 150.6, 145.6, 143.8, 141.8, 139.5, 133.8, 131.3, 130.0, 129.1, 128.3, 128.2 128.0, 127.8, 127.6, 123.2, 121.8, 115.8, 109.0, 95.0, 85.8, 54.7, 52.3, 21.5; HRMS (ESI) calcd for $\mathrm{C}_{29} \mathrm{H}_{24} \mathrm{NO}_{3} \mathrm{~S}[\mathrm{M}+\mathrm{H}]^{+} 466.1471$, found 466.1469. 
<smiles>Oc1c(Br)c(C#CCBr)c2c(c1Br)COC2</smiles>

o-5a-Br: This compound was prepared using GPB and isolated in 42\% yield after purification by column chromatography. ${ }^{1} \mathrm{H}-\mathrm{NMR}\left(\mathrm{CDCl}_{3}, 500 \mathrm{MHz}\right): \delta 5.45(\mathrm{~s}, 1 \mathrm{H}), 5.17-5.15(\mathrm{~m}, 2 \mathrm{H}), 5.07-5.04$ (m, 2H), 2.86-2.81 (m, 2H), $2.44(\mathrm{t}, 2 \mathrm{H}, J=6.8 \mathrm{~Hz}), 1.62-1.36(\mathrm{~m}, 8 \mathrm{H}), 0.95(\mathrm{t}, 3 \mathrm{H}, J=7.3 \mathrm{~Hz}), 0.94$ $(\mathrm{t}, 3 \mathrm{H}, J=7.3 \mathrm{~Hz}) ;{ }^{13} \mathrm{C}-\mathrm{NMR}\left(\mathrm{CDCl}_{3}, 125 \mathrm{MHz}\right): \delta 149.4,136.7,134.3,131.6,117.6,103.0,98.2$, $76.1,75.5,75.4,31.5,30.8,28.8,22.8,21.9,19.3,14.0,13.6$.<smiles>Oc1c(Br)c(Br)c(C#CBr)c2c1COC2</smiles>

$\boldsymbol{m}$-5a-Br: This compound was prepared using GPB and isolated in 27\% yield after purification by column chromatography. ${ }^{1} \mathrm{H}-\mathrm{NMR}\left(\mathrm{CDCl}_{3}, 500 \mathrm{MHz}\right): \delta 5.77(\mathrm{~s}, 1 \mathrm{H}), 5.14(\mathrm{~m}, 2 \mathrm{H}), 5.09(\mathrm{~m}, 2 \mathrm{H})$, $2.92(\mathrm{t}, 2 \mathrm{H}, J=8.1 \mathrm{~Hz}), 2.44(\mathrm{t}, 2 \mathrm{H}, J=6.9 \mathrm{~Hz}), 1.61-1.40(\mathrm{~m}, 8 \mathrm{H}), 0.99-0.92(\mathrm{~m}, 6 \mathrm{H}) ;{ }^{13} \mathrm{C}-\mathrm{NMR}$ $\left(\mathrm{CDCl}_{3}, 125 \mathrm{MHz}\right): \delta 145.9,144.1,143.5,122.9,111.2,110.3,96.3,76.1,74.5,72.8,34.8,31.3,30.9$, 22.8, 21.9, 19.2, 13.9, 13.6; HRMS (ESI) calcd for $\mathrm{C}_{18} \mathrm{H}_{22} \mathrm{BrO}_{2}[\mathrm{M}-\mathrm{H}]^{+}$349.0803, found 349.0800.<smiles>Oc1c(Br)c(C#CCBr)c2c(c1Br)COC2</smiles>

o-5a-I: This compound was prepared using GPB and isolated in 43\% yield after separation using column chromatography. ${ }^{1} \mathrm{H}-\mathrm{NMR}\left(\mathrm{CDCl}_{3}, 500 \mathrm{MHz}\right): \delta 5.23-5.20(\mathrm{~m}, 3 \mathrm{H}), 4.99-4.96(\mathrm{~m}, 2 \mathrm{H}), 2.85$ $(\mathrm{t}, 2 \mathrm{H}, J=7.7 \mathrm{~Hz}), 2.44(\mathrm{t}, 2 \mathrm{H}, J=6.9 \mathrm{~Hz}), 1.62-1.36(\mathrm{~m}, 8 \mathrm{H}), 0.98-0.92(\mathrm{~m}, 6 \mathrm{H}) ;{ }^{13} \mathrm{C}-\mathrm{NMR}\left(\mathrm{CDCl}_{3}\right.$, $125 \mathrm{MHz}): \delta 151.8,140.8,134.1,130.4,118.7,98.5,78.6,78.3,76.0,75.9,31.5,30.8,29.2,22.8$, 21.9, 19.3, 14.0, 13.6; HRMS (ESI) calcd for $\mathrm{C}_{18} \mathrm{H}_{22} \mathrm{IO}_{2}[\mathrm{M}-\mathrm{H}]^{+} 397.0664$, found 397.0660. 
<smiles>Oc1c(I)c(Br)c(C#CCBr)c2c1COC2</smiles>

m-5a-I: This compound was prepared using method B and isolated in $24 \%$ yield after column chromatographic separation. ${ }^{1} \mathrm{H}-\mathrm{NMR}\left(\mathrm{CDCl}_{3}, 500 \mathrm{MHz}\right): \delta 5.61(\mathrm{~s}, 1 \mathrm{H}), 5.14-5.12(\mathrm{~m}, 2 \mathrm{H}), 5.11-5.09$ (m, 2H), 2.99-2.94 (m, 2H), $2.45(\mathrm{t}, 2 \mathrm{H}, J=6.5 \mathrm{~Hz}), 1.62-1.41(\mathrm{~m}, 8 \mathrm{H}), 1.01-0.91(\mathrm{~m}, 6 \mathrm{H}) ;{ }^{13} \mathrm{C}-\mathrm{NMR}$ $\left(\mathrm{CDCl}_{3}, 125 \mathrm{MHz}\right): \delta 148.2,147.4,144.8,122.1,109.8,96.2,92.0,76.3,74.4,73.0,39.6,31.3,30.9$, 22.9, 21.9, 19.2, 13.9, 13.6; HRMS (ESI) calcd for $\mathrm{C}_{18} \mathrm{H}_{24} \mathrm{IO}_{2}[\mathrm{M}+\mathrm{H}]^{+} 399.0815$, found 399.0803.<smiles>COC(=O)C1(C(C)=O)Cc2c(Br)c(O)c(Br)c(C#CCBr)c2C1</smiles>

o-5b-Br: This compound was prepared using GPB at $120{ }^{\circ} \mathrm{C}$ (overnight) and isolated in $63 \%$ yield after column chromatographic purification. ${ }^{1} \mathrm{H}-\mathrm{NMR}\left(\mathrm{CDCl}_{3}, 500 \mathrm{MHz}\right): \delta 5.38(\mathrm{~s}, 1 \mathrm{H}), 3.77(\mathrm{~s}, 6 \mathrm{H})$, $3.68(\mathrm{~s}, 2 \mathrm{H}), 3.58(\mathrm{~s}, 2 \mathrm{H}), 2.80(\mathrm{t}, 2 \mathrm{H}, J=8.0 \mathrm{~Hz}), 2.46(\mathrm{t}, 2 \mathrm{H}, J=7.0 \mathrm{~Hz}), 1.64-1.34(\mathrm{~m}, 8 \mathrm{H}), 0.96$ (t, $3 \mathrm{H}, J=7.5 \mathrm{~Hz}), 0.93(\mathrm{t}, 3 \mathrm{H}, J=8.0 \mathrm{~Hz}) ;{ }^{13} \mathrm{C}-\mathrm{NMR}\left(\mathrm{CDCl}_{3}, 125 \mathrm{MHz}\right): \delta 171.9,149.1,137.1$, 134.7, 131.1, 120.2, 106.6, 98.2, 76.7, 53.1, 42.6, 41.5, 31.5, 30.9, 29.0, 22.8, 22.0, 19.4, 14.0, 13.6; HRMS (ESI) calcd for $\mathrm{C}_{23} \mathrm{H}_{30} \mathrm{BrO}_{5}[\mathrm{M}+\mathrm{H}]^{+} 465.1271$, found 465.1184 .<smiles>Oc1c(Br)c2c(c(C#Cc3ccccc3)c1-c1ccccc1)CN([Te])C2</smiles>

o-5e-Br: This compound was prepared using GPB and isolated in inseparable mix with $36 \%$ overall yield after isolation using column chromatography. ${ }^{1} \mathrm{H}-\mathrm{NMR}\left(\mathrm{CDCl}_{3}, 500 \mathrm{MHz}\right): \delta 7.85-7.82(\mathrm{~m}, 2 \mathrm{H})$, 7.48-7.42 (m, 3H), 7.37-7.33 (m, 2H), 7.31-7.27 (m, 3H), 7.26-7.22 (m, 2H), 7.11-7.08 (m, 2H), 5.94 (s, 1H), 4.79-4.76 (m, 2H), 4.74-4.71 (m, 2H), $2.43(\mathrm{~s}, 3 \mathrm{H}) ;{ }^{13} \mathrm{C}-\mathrm{NMR}\left(\mathrm{CDCl}_{3}, 125 \mathrm{MHz}\right): \delta$ (all discernible signals for both isomers) 148.0, 147.9, 147.6, 147.3, 145.3, 143.9, 143.9, 139.9, 139.3, 138.1, 136.4, 133.8, 131.3, 130.0, 129.6, 129.4, 129.4, 129.2, 129.0, 128.9, 128.8, $128.6,128.4,128.3$, $128.3,128.2,128.0,127.9,127.8,127.6,122.8,122.6,122.3,122.1,111.7,111.0,96.0,84.9,54.5$, 53.4, 52.5, 52.3, 21.5; HRMS (ESI) calcd for $\mathrm{C}_{29} \mathrm{H}_{23} \mathrm{BrNO}_{3} \mathrm{~S}[\mathrm{M}+\mathrm{H}]^{+}$544.0577, found 544.0576. 
<smiles>Oc1c([Al])c(C#CC#[W])c2c(c1Br)C[As]C2</smiles>

o-5f-Br: This compound was prepared using GPB and isolated in $61 \%$ yield after purification by column chromatography. ${ }^{1} \mathrm{H}-\mathrm{NMR}\left(\mathrm{CDCl}_{3}, 500 \mathrm{MHz}\right): \delta$ 7.79-7.76 (m, 2H), 734-7.31 (m, 2H), $5.41(\mathrm{~s}, 1 \mathrm{H})$, 4.67-4.64 (m, 2H), 4.56-4.54 (m, 2H), 2.79-2.74 (m, 2H), 2.44 (t, 2H, J= 7.0 Hz), 2.41 (s, 3H), 1.64-1.57 $(\mathrm{m}, 2 \mathrm{H}), 1.54-1.42(\mathrm{~m}, 4 \mathrm{H}), 1.38-1.25(\mathrm{~m}, 10 \mathrm{H}), 0.92(\mathrm{~m}, 3 \mathrm{H}), 0.90-0.85(\mathrm{~m}, 3 \mathrm{H}) ;{ }^{13} \mathrm{C}-\mathrm{NMR}\left(\mathrm{CDCl}_{3}\right.$, $125 \mathrm{MHz}): \delta 149.7,143.7,133.9,133.7,132.1,131.2,129.9,127.6,119.1,104.2,99.3,75.6,55.7$, 55.0, 31.7, 31.4, 29.4, 29.12, 29.09, 28.7, 28.6, 22.6, 21.5, 19.6, 14.1; HRMS (ESI) calcd for $\mathrm{C}_{29} \mathrm{H}_{39} \mathrm{BrNO}_{3} \mathrm{~S}[\mathrm{M}+\mathrm{H}]^{+} 560.1829$, found 560.1829 .

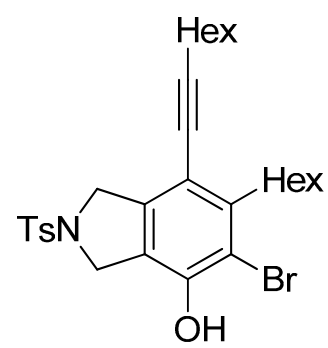

$\boldsymbol{m}$-5f-Br: This compound was prepared using method GPB and isolated in 22\% yield after purification by column chromatography. ${ }^{1} \mathrm{H}-\mathrm{NMR}\left(\mathrm{CDCl}_{3}, 500 \mathrm{MHz}\right): \delta 7.79-7.76(\mathrm{~m}, 2 \mathrm{H}), 7.33-7.30(\mathrm{~m}, 2 \mathrm{H})$, $5.74(\mathrm{~s}, 1 \mathrm{H}), 4.60(\mathrm{~s}, 4 \mathrm{H}), 2.88-2.83(\mathrm{~m}, 2 \mathrm{H}), 2.46-2.42(\mathrm{~m}, 2 \mathrm{H}), 2.41(\mathrm{~s}, 3 \mathrm{H}), 1.63-1.54(\mathrm{t}, 3 \mathrm{H})$, 1.53-1.42 (m, 3H), 1.41-1.27 (m, 10H), 0.94-0.86 (m, 6H); ${ }^{13} \mathrm{C}-\mathrm{NMR}\left(\mathrm{CDCl}_{3}, 125 \mathrm{MHz}\right): \delta 146.5$, 144.6, 143.7, 140.0, 133.9, 129.9, 127.6, 120.2, 111.8, 111.4, 97.3, 75.6, 54.5, 52.4, 35.2, 31.6, 31.4, 29.4, 29.0, 28.8, 28.6, 22.6, 21.5, 19.6, 14.1; HRMS (ESI) calcd for $\mathrm{C}_{29} \mathrm{H}_{39} \mathrm{BrNO}_{3} \mathrm{~S}[\mathrm{M}+\mathrm{H}]^{+}$ 560.1829 , found 560.1829 .

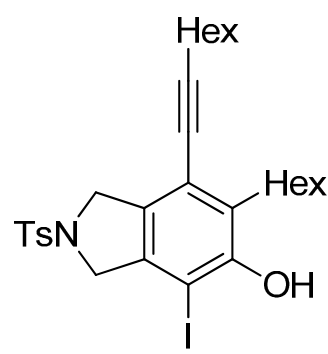

o-5f-I: This compound was prepared using GPB and isolated in 64\% yield after purification by column chromatography. ${ }^{1} \mathrm{H}-\mathrm{NMR}\left(\mathrm{CDCl}_{3}, 500 \mathrm{MHz}\right): \delta 7.80-7.76(\mathrm{~m}, 2 \mathrm{H}), 7.34-7.31(\mathrm{~m}, 2 \mathrm{H}), 5.19(\mathrm{~s}, 1 \mathrm{H})$, 4.71-4.69 (m, 2H), 4.50-4.47 (m, 2H), 2.81-2.76 (m, 2H), $2.44(\mathrm{t}, 2 \mathrm{H}, J=7.0 \mathrm{~Hz}), 2.41(\mathrm{~s}, 3 \mathrm{H})$, 1.64-1.42 (m, 6H), 1.38-1.24 (m, 10H), $0.92(\mathrm{t}, 3 \mathrm{H}, J=6.8 \mathrm{~Hz}), 0.89-0.85(\mathrm{~m}, 3 \mathrm{H}) ;{ }^{13} \mathrm{C}-\mathrm{NMR}$ $\left(\mathrm{CDCl}_{3}, 125 \mathrm{MHz}\right): \delta 152.2,143.7,137.8,133.9,131.0,130.9,130.0,129.9,127.6,120.1,99.6,80.3$, 75.5, 59.1, 55.4, 31.7, 31.4, 29.7, 29.5, 29.4, 29.1, 28.7, 28.6, 22.6, 21.5, 19.6, 14.1; HRMS (ESI) calcd for $\mathrm{C}_{29} \mathrm{H}_{39} \mathrm{INO}_{3} \mathrm{~S}[\mathrm{M}+\mathrm{H}]^{+}$608.1690, found 608.1694 . 


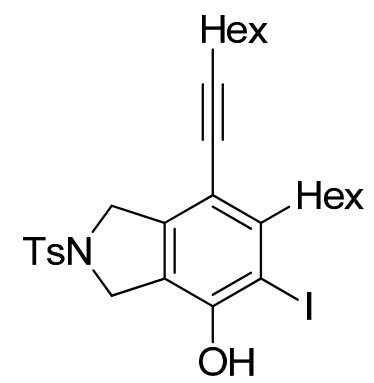

$\boldsymbol{m}$-5f-I: This compound was isolated in 24\% yield using GPB after column chromatographic separation. ${ }^{1} \mathrm{H}-\mathrm{NMR}\left(\mathrm{CDCl}_{3}, 500 \mathrm{MHz}\right): \delta$ 7.79-7.76 (m, 2H), 7.33-7.30 (m, 2H), $5.58(\mathrm{~s}, 1 \mathrm{H}), 4.63-4.59(\mathrm{~m}$, $4 \mathrm{H}), 2.92-2.87(\mathrm{~m}, 2 \mathrm{H}), 2.44(\mathrm{t}, 2 \mathrm{H}, J=7.2 \mathrm{~Hz}), 2.40$ (s, 3H), 1.63-1.29 (m, 16H), 0.94-0.87 (m, 6H); ${ }^{13} \mathrm{C}-\mathrm{NMR}\left(\mathrm{CDCl}_{3}, 125 \mathrm{MHz}\right): \delta 148.9,147.9,143.7,141.3,133.9,129.9,127.6,119.3,111.2,97.2$, $91.3,75.8,54.5,52.7,40.1,31.5,31.4,29.4,29.0,28.8,28.6,22.6,21.5,19.6,14.1$; HRMS (ESI) calcd for $\mathrm{C}_{29} \mathrm{H}_{39} \mathrm{INO}_{3} \mathrm{~S}[\mathrm{M}+\mathrm{H}]^{+} 608.1690$, found 608.1692 .<smiles>CCCC#Cc1c2c(c(Br)c(OC(F)(F)F)c1[Si]CC)C[13C](CC)C2</smiles>

$\boldsymbol{o}-\mathbf{6 g}-\mathbf{C F}_{3}$ : This compound was prepared using GPB and isolated in $72 \%$ yield after column chromatographic purification. ${ }^{1} \mathrm{H}-\mathrm{NMR}\left(\mathrm{CDCl}_{3}, 500 \mathrm{MHz}\right): \delta 7.78-7.75(\mathrm{~m}, 2 \mathrm{H}), 7.36-7.32(\mathrm{~m}, 2 \mathrm{H})$, $4.79-4.58(\mathrm{~m}, 4 \mathrm{H}), 2.42(\mathrm{~s}, 3 \mathrm{H}), 1.08-0.95(\mathrm{~m}, 12 \mathrm{H}), 0.91-0.86(\mathrm{~m}, 12 \mathrm{H}), 0.71(\mathrm{q}, 6 \mathrm{H}, J=7.9 \mathrm{~Hz})$; ${ }^{13} \mathrm{C}-\mathrm{NMR}\left(\mathrm{CDCl}_{3}, 125 \mathrm{MHz}\right): \delta 156.2(\mathrm{C}=\mathrm{O}), 155.8(\mathrm{C}=\mathrm{O}), 155.5(\mathrm{C}=\mathrm{O}), 155.1(\mathrm{C}=\mathrm{O}), 151.0,144.1$, 141.3, 140.2, 133.5, 133.1, 130.0, 127.6, 124.5, $115.6\left(\mathrm{CF}_{3}\right), 113.4\left(\mathrm{CF}_{3}\right), 111.2,104.8,102.3,56.2,55.4$, 21.5, 7.4, 7.3, 4.1, 4.0; HRMS (ESI) calcd for $\mathrm{C}_{31} \mathrm{H}_{42} \mathrm{BrF}_{3} \mathrm{NO}_{4} \mathrm{SSi}_{2}[\mathrm{M}+\mathrm{H}]^{+}$716.1503, found 716.1504.

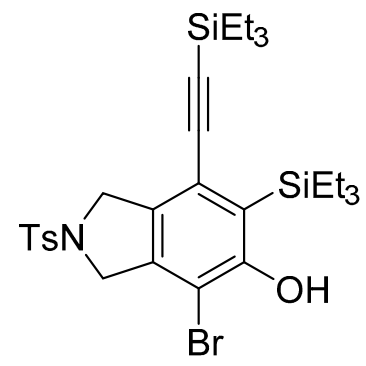

o-5g-Br: This compound was isolated in 10\% after column chromatographic purification in GPB. ${ }^{1} \mathrm{H}-\mathrm{NMR}\left(\mathrm{CDCl}_{3}, 500 \mathrm{MHz}\right): \delta$ 7.77-7.74 (m, 2H), 7.34-7.31 (m, 2H), $5.64(\mathrm{~s}, 1 \mathrm{H}), 4.64-4.58(\mathrm{~m}, 4 \mathrm{H})$, $2.41(\mathrm{~s}, 3 \mathrm{H}), 1.07-0.95(\mathrm{~m}, 15 \mathrm{H}), 0.94-0.89(\mathrm{~m}, 9 \mathrm{H}), 0.69(\mathrm{q}, 6 \mathrm{H}, J=6.9 \mathrm{~Hz})$. HRMS (ESI) calcd for $\mathrm{C}_{29} \mathrm{H}_{43} \mathrm{BrNO}_{3} \mathrm{SSi}_{2}[\mathrm{M}+\mathrm{H}]^{+}$620.1680, found 620.1669 . 


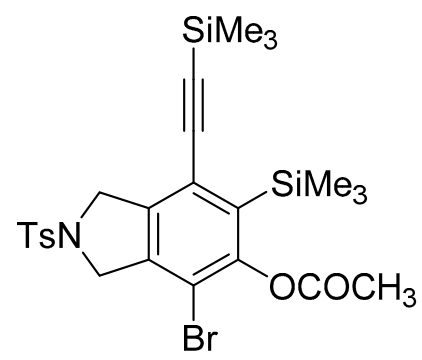

o-6d-CH3: This compound was prepared using GPB and isolated in $16 \%$ yield after purification by column chromatography. ${ }^{1} \mathrm{H}-\mathrm{NMR}\left(\mathrm{CDCl}_{3}, 500 \mathrm{MHz}\right): \delta$ 7.79-7.76 $(\mathrm{m}, 2 \mathrm{H}), 7.36-7.33(\mathrm{~m}, 2 \mathrm{H})$, $4.8-4.53(\mathrm{~m}, 4 \mathrm{H}), 2.43(\mathrm{~s}, 3 \mathrm{H}), 2.31(\mathrm{~s}, 3 \mathrm{H}), 0.36(\mathrm{~s}, 9 \mathrm{H}), 0.25(\mathrm{~s}, 9 \mathrm{H}) ;{ }^{13} \mathrm{C}-\mathrm{NMR}\left(\mathrm{CDCl}_{3}, 125 \mathrm{MHz}\right)$ : $\delta 169.0,151.8,144.0,139.5,139.2,135.8,133.6,130.0,127.5,123.6,112.7,105.8,101.7,56.1,55.1$, 21.5, 21.3, 0.7, -0.4; HRMS (ESI) calcd for $\mathrm{C}_{25} \mathrm{H}_{33} \mathrm{BrNO}_{4} \mathrm{SSi}_{2}[\mathrm{M}+\mathrm{H}]^{+}$578.0847, found 578.0846.<smiles>CSC#Cc1c2c(c(Br)c(O)c1SC)CN([As])C2</smiles>

o-5d-Br: This compound was prepared using GPB and isolated in 36\% yield after purification by column chromatography. ${ }^{1} \mathrm{H}-\mathrm{NMR}\left(\mathrm{CDCl}_{3}, 500 \mathrm{MHz}\right): \delta$ 7.79-7.76 (m, 2H), 7.35-7.32 (m, 2H), $5.66(\mathrm{~s}, 1 \mathrm{H})$, 4.66-4.64 (m, 2H), 4.58-4.56 (m, 2H), $2.42(\mathrm{~s}, 3 \mathrm{H}), 0.38(\mathrm{~s}, 9 \mathrm{H}), 0.25(\mathrm{~s}, 9 \mathrm{H}) ;{ }^{13} \mathrm{C}-\mathrm{NMR}\left(\mathrm{CDCl}_{3}\right.$, $125 \mathrm{MHz}): \delta 155.9,143.8,137.9,133.8,133.5,129.9,127.5,127.5,123.5,105.8,104.1,102.2,56.0$, 55.0, 21.5, 1.1, -0.4; HRMS (ESI) calcd for $\mathrm{C}_{23} \mathrm{H}_{31} \mathrm{BrNO}_{3} \mathrm{SSi}_{2}[\mathrm{M}+\mathrm{H}]^{+}$536.0741, found 536.0740.<smiles>CSc1c(C#C[As]C)c2c(c(I)c1OC(F)(F)F)CN([Te])C2</smiles>

o-6d: This compound was prepared using GPB and isolated in $48 \%$ yield after purification by column chromatography. ${ }^{1} \mathrm{H}-\mathrm{NMR}\left(\mathrm{CDCl}_{3}, 500 \mathrm{MHz}\right): \delta$ 7.80-7.77 (m, 2H), 7.37-7.34 (m, 2H), 4.86-4.68 $(\mathrm{m}, 2 \mathrm{H}), 4.63-4.49(\mathrm{~m}, 2 \mathrm{H}), 2.43(\mathrm{~s}, 3 \mathrm{H}), 0.36(\mathrm{~s}, 9 \mathrm{H}), 0.26(\mathrm{~s}, 9 \mathrm{H}) ;{ }^{13} \mathrm{C}-\mathrm{NMR}\left(\mathrm{CDCl}_{3}, 125 \mathrm{MHz}\right): \delta$ $156.0(\mathrm{C}=\mathrm{O}), 155.7(\mathrm{C}=\mathrm{O}), 152.9,144.7,144.1,139.7,134.7,133.6,130.1,127.5,125.2,118.0\left(\mathrm{CF}_{3}\right)$, $115.7\left(\mathrm{CF}_{3}\right), 113.5\left(\mathrm{CF}_{3}\right), 107.2,101.2,86.4,59.7,55.4,21.5,0.6,-0.5$; HRMS (ESI) calcd for $\mathrm{C}_{25} \mathrm{H}_{30} \mathrm{~F}_{3} \mathrm{INO}_{4} \mathrm{SSi}_{2}[\mathrm{M}+\mathrm{H}]^{+} 680.0425$, found 680.0433 . 
<smiles>Brc1c(Br)c(C(Br)(Br)Br)c(Br)c2c1COC2</smiles>

o-6a: This compound was prepared using method GPB in an inseparable mixture of two isomers (2.7:1) with $83 \%$ overall yield after column chromatography purification. ${ }^{1} \mathrm{H}-\mathrm{NMR}\left(\mathrm{CDCl}_{3}, 500 \mathrm{MHz}\right)$ : $\delta$ (major isomer) 5.22-5.20 (m, 2H), 5.11-5.09 (m, 2H), 2.94-2.90 (m, 2H), $2.46(\mathrm{t}, 2 \mathrm{H}, J=6.5 \mathrm{~Hz})$, 1.64-1.36 (m, 8H), 0.98-0.92 (m, 6H); ${ }^{13} \mathrm{C}-\mathrm{NMR}\left(\mathrm{CDCl}_{3}, 500 \mathrm{MHz}\right): \delta$ (all discernible signals for both isomers) $146.7,144.6,143.9,142.8,139.8,139.6,139.4,130.9,130.1,129.9,129.7,129.6,128.2$, $128.1,127.9,126.0,125.6,119.9,118.8,118.6,117.4,116.8,108.9,100.7,100.4,75.8,75.3,74.5$, 72.8, 35.1, 35.1, 31.6, 31.0, 30.6, 30.6, 29.8, 22.8, 22.7, 21.9, 19.3, 19.2, 13.8, 13.7, 13.5; HRMS (ESI) calcd for $\mathrm{C}_{19} \mathrm{H}_{21} \mathrm{BrF}_{3} \mathrm{O}_{4} \mathrm{~S}[\mathrm{M}-\mathrm{H}]^{+}$481.0296, found 481.0305 .

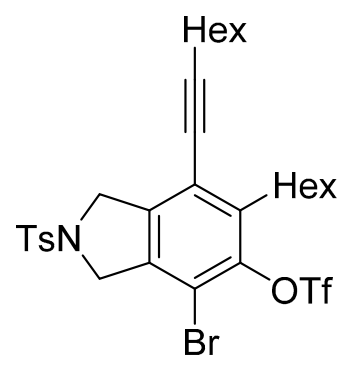

o-6f: This compound was prepared using GPB and isolated in $78 \%$ yield after purification by column chromatography. ${ }^{1} \mathrm{H}-\mathrm{NMR}\left(\mathrm{CDCl}_{3}, 500 \mathrm{MHz}\right): \delta 7.80-7.76(\mathrm{~m}, 2 \mathrm{H}), 7.36-7.33(\mathrm{~m}, 2 \mathrm{H}), 4.71(\mathrm{~s}, 2 \mathrm{H})$, $4.60(\mathrm{~s}, 2 \mathrm{H}), 2.87-2.83(\mathrm{~m}, 2 \mathrm{H}), 2.46(\mathrm{t}, 2 \mathrm{H}, J=7.0 \mathrm{~Hz}), 2.42(\mathrm{~s}, 3 \mathrm{H}), 1.65-1.42(\mathrm{~m}, 6 \mathrm{H}), 1.38-1.25$ $(\mathrm{m}, 10 \mathrm{H}), 0.95-0.91(\mathrm{~m}, 3 \mathrm{H}), 0.90-0.86(\mathrm{~m}, 3 \mathrm{H}) ;{ }^{13} \mathrm{C}-\mathrm{NMR}\left(\mathrm{CDCl}_{3}, 125 \mathrm{MHz}\right): \delta 144.1,144.1,141.0$, 139.7, 136.9, 133.6, 130.0, 127.5, $122.4\left(\mathrm{CF}_{3}\right), 120.4,119.8\left(\mathrm{CF}_{3}\right), 117.3\left(\mathrm{CF}_{3}\right), 110.5,101.9,74.8$, 56.0, 55.1, 31.4, 31.3, 30.2, 29.4, 29.3, 28.6, 28.5, 22.6, 22.5, 21.5, 19.7, 14.1, 14.0; HRMS (ESI) calcd for $\mathrm{C}_{30} \mathrm{H}_{38} \mathrm{BrF}_{3} \mathrm{NO}_{5} \mathrm{~S}_{2}[\mathrm{M}+\mathrm{H}]^{+}$692.1321, found 692.1309 .

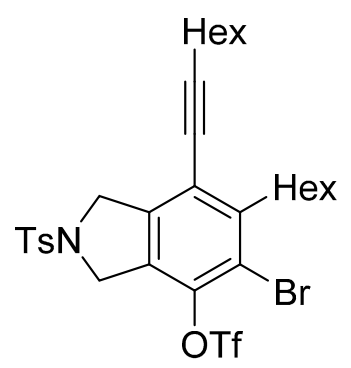

m-6f: This compound was isolated as minor isomer in an inseparable mix with previous compound with $16 \%$ yield after purification using column chromatography. ${ }^{1} \mathrm{H}-\mathrm{NMR}\left(\mathrm{CDCl}_{3}, 500 \mathrm{MHz}\right): \delta 7.78-7.75$ $(\mathrm{m}, 2 \mathrm{H}), 7.35-7.32(\mathrm{~m}, 2 \mathrm{H}), 4.73(\mathrm{~s}, 2 \mathrm{H}), 4.61(\mathrm{~s}, 2 \mathrm{H}), 2.96-2.91(\mathrm{~m}, 2 \mathrm{H}), 2.48(\mathrm{t}, 2 \mathrm{H}, J=7.1 \mathrm{~Hz})$, $2.41(\mathrm{~s}, 3 \mathrm{H}), 1.65-1.28(\mathrm{~m}, 16 \mathrm{H}), 0.95-0.86(\mathrm{~m}, 6 \mathrm{H})$; HRMS (ESI) calcd for $\mathrm{C}_{30} \mathrm{H}_{3} \mathrm{BrF}_{3} \mathrm{NO}_{5} \mathrm{~S}_{2}$ $[\mathrm{M}+\mathrm{H}]^{+}$692.1321, found 692.1306 . 


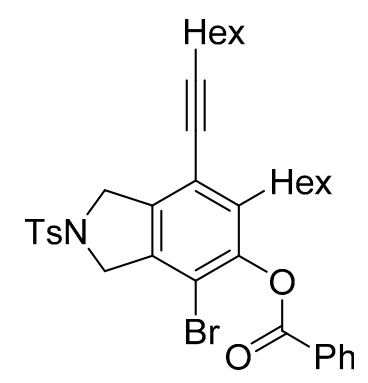

o-6f-Br: This compound was formed by GPB in an inseparable mixture of two isomers (ratio 2:1) with $30 \%$ overall yield after column chromatography. ${ }^{1} \mathrm{H}-\mathrm{NMR}\left(\mathrm{CDCl}_{3}, 500 \mathrm{MHz}\right): \delta$ (major isomer) $8.22-8.19$ $(\mathrm{m}, 2 \mathrm{H}), 7.81-7.77(\mathrm{~m}, 2 \mathrm{H}), 7.57-7.51(\mathrm{~m}, 3 \mathrm{H}), 7.37-7.33(\mathrm{~m}, 2 \mathrm{H}), 4.65-4.62(\mathrm{~m}, 2 \mathrm{H}), 4.59-4.54$ (m, 2H), 2.56-2.38 (m, 7H), 1.66-1.18 (m, 16H) , 0.96-0.87 (m, 6H); ${ }^{13} \mathrm{C}-\mathrm{NMR}\left(\mathrm{CDCl}_{3}, 125 \mathrm{MHz}\right): \delta$ (all discernible signals for both isomers) 164.0, 163.3, 146.4, 145.89, 143.92, 143.84, 139.8, 138.9, $137.4,135.4$, 135.4, 134.5, 134.2, 134.0, 133.64, 130.6, 130.5, 130.3, 130.0, 129.96, 128.9, 128.8, $128.7,127.6,127.5,119.2,117.8,111.0,100.3,99.6,75.6,75.3,55.8,55.2,54.4,52.7,35.2,31.6$, $31.4,31.3,29.7,29.5,29.3,29.2,28.9,28.7,28.6,22.6,22.5,21.5,19.7,19.6,14.1,14.0$; HRMS (ESI) calcd for $\mathrm{C}_{36} \mathrm{H}_{43} \mathrm{BrNO}_{4} \mathrm{~S}[\mathrm{M}+\mathrm{H}]^{+}$664.2091, found 664.2079.

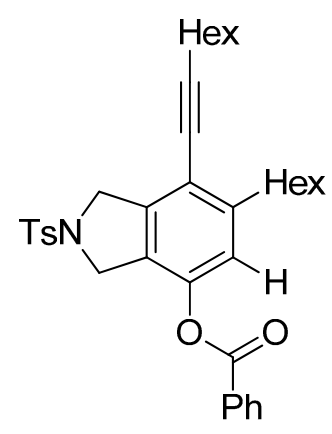

o-6f-H: This compound was isolated in $24.5 \%$ using GPB after purification by column chromatography. ${ }^{1} \mathrm{H}-\mathrm{NMR}\left(\mathrm{CDCl}_{3}, 500 \mathrm{MHz}\right): \delta 8.17-8.13(\mathrm{~m}, 2 \mathrm{H}), 7.78-7.74(\mathrm{~m}, 2 \mathrm{H}), 7.69-7.64(\mathrm{~m}, 1 \mathrm{H}), 7.56-7.50$ $(\mathrm{m}, 2 \mathrm{H}), 7.34-7.29(\mathrm{~m}, 2 \mathrm{H}), 6.94(\mathrm{~s}, 1 \mathrm{H}), 4.66(\mathrm{~s}, 2 \mathrm{H}), 4.57(\mathrm{~s}, 2 \mathrm{H}), 2.75-2.68(\mathrm{~m}, 2 \mathrm{H}), 2.50-2.44$ $(\mathrm{m}, 2 \mathrm{H}), 2.41(\mathrm{~s}, 3 \mathrm{H}), 1.66-1.53(\mathrm{~m}, 4 \mathrm{H}), 1.52-1.44(\mathrm{~m}, 2 \mathrm{H}), 1.39-1.24(\mathrm{~m}, 10 \mathrm{H}), 0.97-0.91(\mathrm{~m}, 3 \mathrm{H})$, 0.90-0.85 (m, 3H); ${ }^{13} \mathrm{C}-\mathrm{NMR}\left(\mathrm{CDCl}_{3}, 125 \mathrm{MHz}\right) \delta 164.1,146.6,144.4,143.7,141.0,134.0,133.9$, 130.3, 129.9, 128.8, 128.7, 127.6, 125.8, 121.2, 116.4, 98.8, 75.6, 54.5, 52.4, 34.2, 31.7, 31.4, 30.5, 29.7, 29.1, 28.8, 28.6, 22.6, 22.6, 21.5, 19.6, 14.1; HRMS (ESI) calcd for $\mathrm{C}_{36} \mathrm{H}_{44} \mathrm{NO}_{4} \mathrm{~S}[\mathrm{M}+\mathrm{H}]^{+}$ 586.2986 , found 586.2990 .<smiles>N#Cc1c(OC(=O)c2ccccc2)cc2c(c1C#CCCl)CN([Te])C2</smiles>

m-6f-H: This compound was prepared using GPB and isolated in 51.5\% yield (with $85 \%$ purity) after purification by column chromatography. ${ }^{1} \mathrm{H}-\mathrm{NMR}\left(\mathrm{CDCl}_{3}, 500 \mathrm{MHz}\right): \delta 8.18-8.15(\mathrm{~m}, 2 \mathrm{H}), 7.80-7.76$ 
(m, 2H), 7.67-7.62 (m, 1H), 7.54-7.49 (m, 2H), 7.35-7.30 (m, 2H), $6.88(\mathrm{~s}, 1 \mathrm{H}), 4.64(\mathrm{~s}, 2 \mathrm{H}), 4.62$ (s, 2H), 2.69-2.64 (m, 2H), 2.49-2.44 (m, 2H), $2.42(\mathrm{~s}, 3 \mathrm{H}), 1.65-1.57(\mathrm{~m}, 2 \mathrm{H}), 1.54-1.42(\mathrm{~m}, 4 \mathrm{H})$, $1.38-1.17(\mathrm{~m}, 10 \mathrm{H}), 0.94-0.90(\mathrm{~m}, 3 \mathrm{H}), 0.81-0.76(\mathrm{~m}, 3 \mathrm{H}) ;{ }^{13} \mathrm{C}-\mathrm{NMR}\left(\mathrm{CDCl}_{3}, 125 \mathrm{MHz}\right): \delta 165.2$, $148.8,143.7,136.6,136.5,134.0,133.8,133.7,130.1,129.9,129.2,128.7,127.6,120.4,116.1,99.6$, $75.8,54.2,54.1,31.5,31.4,29.6,29.3,28.8,28.7,28.6,22.6,22.5,21.5,19.6,14.1,14.0$.

\section{Conclusions}

In conclusion, we developed a formal hydration method of arynes generated from hexadehydro Diels-Alder reaction. While direct use of water does not efficiently trap the in situ generated arynes to generate phenolic products, silver trifluroacetate $\left(\mathrm{AgO}_{2} \mathrm{CCF}_{3}\right)$ can behave as an effective water surrogate in these reactions. This is probably due to the improved miscibility and reactivity of $\mathrm{AgO}_{2} \mathrm{CCF}_{3}$ with arynes, compared to water, to generate the corresponding trifluoroacetoxy organosilver arene intermediates, and, upon treating, with silica gel, these intermediates readily undergo protonolysis of their carbon-silver bonds and hydrolysis of the trifluoroacetyl groups. This protocol can be extended to the synthesis of $\alpha$-halofunctionalized phenol derivatives by simply adding NBS or NIS to the reaction along with silver trifluroacetate, which provided $\alpha$-bromo or $\alpha$-iodophenol derivatives in good yield. Interestingly, the similar reactions with NCS afforded only the corresponding protonated products instead of the expected $\alpha$-chlorophenols derivatives. Unexpectedly, reactions of substrates containing trialkylsilyl substituents on 1,3-diynes provided $\alpha$-halotrifluoroacetates rather than their hydrolyzed products. Trapping the same arynes with other oxygen-based nucleophiles containing a silver counter cation, along with NXS, generated $\alpha$-halooxyfunctionalized products in good yields.

\section{Supplementary Materials}

Supplementary materials can be accessed at: http://www.mdpi.com/1420-3049/20/09/15862/s1.

\section{Acknowledgments}

Financial support from UIC (LAS AFS) and the National Science Foundation (CHE 1361620) is greatly acknowledged. We are grateful to Furong Sun of the University of Illinois at Urbana-Champaign for high resolution mass spectrometry data.

\section{Author Contributions}

D.L. designed the research and wrote the paper. R.K. and S.G. performed the bench work for synthesizing starting materials and products. Everyone contributed to the analysis of the spectra. Y.X. carried out the computational study. All authors read and approved the final manuscript.

\section{Conflicts of Interest}

The authors declare no conflict of interest. 


\section{References and Notes}

1. Pellissier, H.; Santelli, M. The Use of Arynes in Organic Synthesis. Tetrahedron 2003, 59, 701-730.

2. Dyke, A.M.; Hester, A.J.; Lloyd-Jones, G.C. Organometallic Generation and Capture of ortho-Arynes. Synthesis 2006, 24, 4093-4112.

3. Sanz, R. Recent Applications of Aryne Chemistry to Organic Synthesis. A Review. Org. Prep. Proced. Int. 2008, 40, 215-291.

4. Chen, Y.; Larock, R.C. Arylation Reactions Involving the formation of arynes. In Modern Arylation Methods; Akermann, L., Ed.; WILEY-VCH: Weinheim, Germany, 2009; pp. 401-473.

5. Kitamura, T. Synthetic Methods for the Generation and Preparative Application of Benzyne. Aust. J. Chem. 2010, 63, 987-1001.

6. Tadross, P.M.; Stoltz, B.M. A Comprehensive History of Arynes in Natural Product Total Synthesis. Chem. Rev. 2012, 112, 3550-3577.

7. Gampe, C.M.; Carreira, E.M. Arynes and Cyclohexyne in Natural Product Synthesis. Angew. Chem. Int. Ed. 2012, 51, 3766-3778.

8. Wu, C.; Shi, F. A Closer Look at Aryne Chemistry: Details that Remain Mysterious. Asian J. Org. Chem. 2013, 2, 116-125.

9. Rodan, N.G.; Domelsmith, L.N.; Houk, K.N. The Relative Rates of Electron-Rich and Electron-Deficient Alkene Cycloadditions to Benzyne. Enhanced Electrophilicity as a Consequence of Alkyne Bending Distortion. Tetrahedron Lett. 1979, 20, 3237-3240.

10. Kitamura, T.; Yamane, M. (Phenyl)[o-(Trimethylsilyl)phenyl]iodonium Triflate. A New and Efficient Precursor of Benzyne. J. Chem. Soc. Chem. Commun. 1995, 983-984, doi:10.1039/C39950000983.

11. Himeshima, Y.; Sonoda, T.; Kobayashi, H. Fluoride-Induced 1,2-Elimination of o-Trimethylsilylphenyl Triflate to Benzyne Under Mild Conditions. Chem. Lett. 1983, 12, 1211-1214.

12. Campbell, C.D.; Rees, C.W. Reactive Intermediates. Part 1. Synthesis and Oxidation of 1- and 2-Aminobenzotriazole. J. Chem. Soc. C 1969, 742-747.

13. Matsumoto, T.; Hosoya, T.; Katsuki, M.; Suzuki, K. New Efficient Protocol for Aryne Generation. Selective Synthesis of Differentially Protected 1,4,5-Naphthalenetriols. Tetrahedron Lett. 1991, 32, 6735-6736.

14. Friedman, L.; Logullo, F.M. Reactions of Benzyne Intermediates in Non-basic Media. J. Am. Chem. Soc. 1963, 85, 1792-1797.

15. Hoffmann, R.W. Dehydrobenzene and Cycloalkynes; Verlag Chemie-Academic Press: New York, NY, USA, 1967.

16. Wittig, G.; Hoffmann, R.W. 1,2,3-Benzothiadiazole 1,1-dioxide. Org. Synth. 1967, 47, doi:10.1002/0471264180.os047.01.

17. Miyawaki, K.; Suzuki, R.; Kawano, T.; Ueda, I. Cycloaromatization of a Non-Conjugated Polyenyne System: Synthesis of $5 H$-Benzo[d]fluoreno[3,2-b]pyrans via Diradicals Generated from 1-[2-\{4(2-Alkoxymethylphenyl)butan-1,3-diynyl\}] phenylpentan-2,4-diyn.1-ols and Trapping Evidence for the 1,2-Didehydrobenzene Diradical. Tetrahedron Lett. 1997, 38, 3943-3946.

18. Bradley, A.Z.; Johnson, R.P. Thermolysis of 1,3,8-Nonatriyne: Evidence for Intramolecular [2+4] Cycloaromatization to a Benzyne Intermediate. J. Am. Chem. Soc. 1997, 119, 9917-9918. 
19. Hoye, T.R.; Baire, B.; Niu, D.; Willoughby, P.H.; Woods, B.P. The Hexadehydro-Diels-Alder Reaction. Nature 2012, 490, 208-212.

20. Yun, S.Y.; Wang, K.P.; Lee, N.K.; Mamidipalli, P.; Lee, D. Alkane C-H Insertion by Aryne Intermediates with a Silver Catalyst. J. Am. Chem. Soc. 2013, 135, 4668-4671.

21. Karmakar, R.; Yun, S.Y.; Wang, K.P.; Lee, D. Regioselectivity in the Nucleophile Trapping of Arynes: The Electronic and Steric Effects of Nucleophiles and Substituents. Org. Lett. 2014, 16, 6-9.

22. Willoughby, P.H.; Niu, D.; Wang, T.; Haj, M.K.; Cramer, C.J.; Hoye, T.R. Mechanism of the Reactions of Alcohols with o-Benzynes. J. Am. Chem. Soc. 2014, 136, 13657-13665.

23. Whiting, D.A. Natural Phenolic Compounds 1900-2000: A Bird's Eye View of a Century's Chemistry. Nat. Prod. Rep. 2001, 18, 583-606.

24. Martins, S.; Mussatto, S.I.; Martínez-Avila, G.; Montañez-Saenz, J.; Aguilar, C.N.; Teixeira, J.A. Bioactive Phenolic Compounds: Production and Extraction by Solid-State Fermentation. A Review. Biotechnol. Adv. 2011, 29, 365-373.

25. Wang, K.P.; Yun, S.Y.; Mamidipalli, P.; Lee, D. Silver-Mediated Fluorination, Trifluoromethylation and Trifluoromethylthiolation of Arynes. Chem. Sci. 2013, 4, 3205-3211.

26. The reaction needed overnight heating at $120^{\circ} \mathrm{C}$ for complete consumption of the substrate.

27. Bronner, S.M.; Goetz, A.E.; Garg, N.K. Overturning Indolyne Regioselectivities and Synthesis of Indolactam V. J. Am. Chem. Soc. 2011, 133, 3832-3835.

28. Cheong, P.H.Y.; Paton, R.S.; Bronner, S.M.; Im, G.Y.J.; Garg, N.K.; Houk, K.N. Indolyne and Aryne Distortions and Nucleophilic Regioselectivites. J. Am. Chem. Soc. 2010, 132, 1267-1269.

29. Bronner, S.M.; Mackey, J.L.; Houk, K.N.; Garg, N.K. Steric Effects Compete with Aryne Distortion to Control Regioselectivities of Nucleophilic Additions to 3-Silylarynes. J. Am. Chem. Soc. 2012, 134, 13966-13969.

30. Medina, J.M.; Mackey, J.L.; Garg, N.K.; Houk, K.N. The Role of Aryne Distortions, Steric Effects, and Charges in Regioselectivities of Aryne Reactions. J. Am. Chem. Soc. 2014, 136, 15798-15805.

31. Yoshida, H.; Fukushima, H.; Ohshita, J.; Kunai, A. Arynes in a Three-Component Coupling Reaction: Straightforward Synthesis of Benzoannulated Iminofurans. Angew. Chem. Int. Ed. 2004, 43, 3935-3938.

32. Yoshida, H.; Fukushima, H.; Ohshita, J.; Kunai, A. Straightforward Access to 2-Iminoisoindolines via Three-Component Coupling of Arynes, Isocyanides and Imines. Tetrahedron Lett. 2004, 45, 8659-8662.

33. Yoshida, H.; Fukushima, H.; Morishita, T.; Ohshita, J.; Kunai, A. Three-Component Coupling using Arynes and Isocyanides: Straightforward Access to Benzo-Annulated Nitrogen or Oxygen Heterocycles. Tetrahedron 2007, 63, 4793-4805.

34. Allan, K.M.; Gilmore, C.D.; Stoltz, B.M. Benzannulated Bicycles by Three-Component Aryne Reactions. Angew. Chem. Int. Ed. 2011, 50, 4488-4491.

35. Yoshida, H.; Takaki, K. Multicomponent Coupling Reaction of Arynes for Construction of Heterocyclic Skeletons. Heterocycles 2012, 85, 1333-1349.

36. Li, J.; Noyori, S.; Iwasaki, M.; Nakajima, K.; Nishihara, Y. A Novel Three-Component Coupling Reaction of Arynes, Isocyanides, and Cyanoformates: A Straightforward Access to Cyano-Substituted Iminoisobenzofurans. Heterocycles 2012, 86, 933-940. 
37. Li, J.; Noyori, S.; Nakajima, K.; Nishihara, Y. New Entry to the Synthesis of $\alpha$-Iminonitriles by Lewis Acid Mediated Isomerization of Cyano-Substituted Iminoisobenzofurans Prepared by Palladium-Catalyzed Three-Component Coupling of Arynes, Isocyanides, and Cyanoformates. Organometallics 2014, 33, 3500-3507.

38. The observed regioselectivity for the reactions of $\mathbf{1 d}$ and $\mathbf{1 e}$ can be further confirmed by the Mulliken Population analysis (but less accurately by NBO analysis) of aryne intermediates. See Supplementary Materials for the calculations.

39. Regiochemistry of the products were predicted on the basis of product distribution in a reaction of 1f with AgOTf in the absence of any electrophile. The product was formed in 4.4:1 ratio favoring the ortho-addition of triflate.

Sample Availability: Samples of the compounds are not available from the authors.

(C) 2015 by the authors; licensee MDPI, Basel, Switzerland. This article is an open access article distributed under the terms and conditions of the Creative Commons Attribution license (http://creativecommons.org/licenses/by/4.0/). 